\title{
Characterization of terrestrial hot particles from the Palomares accident using destructive and non-destructive analytical techniques
}

\author{
M.C. Jiménez-Ramos ${ }^{1}$, J. García-López ${ }^{1}$, R. García-Tenorio ${ }^{2}$ \\ and M. García-León ${ }^{1}$ \\ ${ }^{1}$ Centro Nacional de Aceleradores, Av. Thomas Alba Edison, Isla de la Cartuja, \\ 41092 Sevilla, Spain \\ ${ }^{2}$ Departamento de Física Aplicada II, E.T.S.A, Universidad de Sevilla, 41012 Sevilla, Spain \\ e-mail: gtenorio@us.es
}

\begin{abstract}
Several radioactive particles from the Palomares area have been analyzed with the nuclear microprobe of the National Accelerator Centre (CNA) in Seville exploring the advantages of combining $\mu$-PIXE using a $5.5 \mathrm{MeV}{ }^{4} \mathrm{He}$ beam with conventional $\mu$-PIXE analysis using $3 \mathrm{MeV}$ protons. The measurements performed with the $\alpha$-particles are more sensitive to the sample surface, while the results collected with protons are indicative of the average composition of the hot particle. In this way, important information about the depth distribution of $\mathrm{Pu}$ and $\mathrm{U}$ in the particles has been obtained. In addition, the particles have been analyzed by SEM (EDX) and one of them has been sacrificed for the determination of its $\mathrm{U}$ and $\mathrm{Pu}$ isotopic composition by ICP-MS and $\alpha$-spectrometry, being gained in this way important information about the composition of the weapons involved in the Palomares accident.
\end{abstract}

\section{INTRODUCTION}

More than 40 years ago, an aircraft accident took place in Palomares (Spain) which involved the destruction of two nuclear weapons and the consequent dispersion of fissile material in a terrestrial area. After clean-up operations, some transuranic contamination in the affected soils remains, being a considerable fraction of this remaining contamination present in the form of small high activity concentration particles, also called "hot particles", mainly formed by plutonium and uranium.

Environmental radioactive hot particles have placed in recent years a very important role for the understanding of the radionuclide transport mechanisms in the environment as well as for their availability to humans. For accurate assessments of their environmental impacts and associated risks is extremely important the characterization of these hot-particles in terms of their morphology, size distribution, elemental and isotopic composition, crystalline structure and oxidation states.

Our group has carried out some previous works regarding to Palomares hot particles isolation, identification and characterization. Average compositional information, mappings and depth distributions of the main elements composing Palomares particles were obtained by a simultaneous combination of micro Particle Induced X-ray Emission ( $\mu$-PIXE) and micro Rutherford Backscattering Spectrometry ( $\mu$-RBS) [1]. Moreover, to obtain information about the $\mathrm{Pu}$ and $\mathrm{U}$ isotopic composition of the particles, radiometric (alpha-particle) and non-radiometric (ICP-MS) techniques were applied in other study [2]. For the application of these last techniques it was necessary to sacrifice a couple of isolated particles but the information obtained ${ }^{238} \mathrm{Pu} /{ }^{239+240} \mathrm{Pu},{ }^{240} \mathrm{Pu} /{ }^{239} \mathrm{Pu},{ }^{235} \mathrm{U} /{ }^{238} \mathrm{U}$ and ${ }^{234} \mathrm{U} /{ }^{238} \mathrm{U}$ activity ratios) together with the previously obtained by the application of the non-destructive analytical techniques ( $\mu$-PIXE, $\mu$-RBS) was essential for gaining information on the contamination source-term, i.e. for assessing the long-term impact from the radioactive particle contamination in the Palomares affected terrestrial area. 
In this work, a comparison between the $\mathrm{Pu} / \mathrm{U}$ elemental ratios obtained by $\mu$-PIXE with protons at $3 \mathrm{MeV}$ and alpha particles at $5.5 \mathrm{MeV}$ and by X-Ray Microscopy Analysis (XRMA) with electrons at $30 \mathrm{keV}$ has been carried out. The results obtained with each beam can be considered as complementary because the average path lengths travelled in the hot particles for the three beams are different. The electrons give superficial information, the alpha particles are more sensitive to the sample surface and the data collected with protons are representative of the whole particle. For that, important conclusions about the homogeneity or heterogeneity of the $\mathrm{Pu} / \mathrm{U}$ elemental ratio in depth for the particles have been gained with this study. In addition, the characterization by ICP-MS and $\alpha$-spectrometry of one isolated particle is shown in order to give information about the composition of the weapons involved in the accident.

\section{MATERIALS AND METHODS}

The hot particles were isolated from soil samples collected in October 2001 at the west of Palomares village, near to the place where one of the bombs exploded. The isolation was carried out by screening superficial soils using gamma-ray spectrometry, through discrimination of the high activity concentrations of ${ }^{241} \mathrm{Am}$ (which indicates the presence of plutonium). The sample splitting process and the sampling campaign are fully described in [3].

\subsection{Scanning Electron Microscopy}

A scanning electron microscope Philips XL-30 with secondary and backscattering electron detectors was used. The hot particles were identified by backscattered electron mode (BSE) (the isolated particles are mainly composed by elements with very high atomic number). Additional information about their morphology and size was gained using SEM in the secondary electron mode (SE).

Information about the superficial composition of the isolated particles was obtained performing XRMA. Indeed, using an EDX spectrometer integrated with an electron microscopy system, pointing $\mathrm{X}$-ray analyses were performed and superficial elemental distributions were obtained through mapping.

The working acceleration voltage was $30 \mathrm{keV}$ to optimize the excitation of the $\mathrm{Pu}$ and $\mathrm{U} \mathrm{L}_{\alpha}$ lines of $14.259 \mathrm{keV}$ and $13.597 \mathrm{keV}$ respectively. By SEM and by XRMA fundamental information was obtained in this work. On one hand, $\mathrm{Pu} / \mathrm{U}$ ratios for different spots in one of the hot particles were determined using a semiquantitative evaluation. On the other, the SEM images were essential to locate the hot particles and to perform the measurements by PIXE.

\subsection{Micro Particle induced X-ray emission}

The elemental analysis by $\mu$-PIXE was carried out using the $3 \mathrm{MV}$ tandem accelerator of the Centro Nacional de Aceleradores (CNA), Seville and a microprobe focussing system. In one experiment, the samples were irradiated with a $3 \mathrm{MeV}$ proton beam, and in other set of measurements, with a 5.5 $\mathrm{MeV} \mathrm{He}^{++}$beam. A retractable Gresham $\mathrm{Si}(\mathrm{Li})$ detector was used, together with a Titan amplifier, for PIXE analysis. The spectra were fitted with WINQXAS program package to deconvolute the PIXE spectra and to determine the area of the $\mathrm{Pu}$ and $\mathrm{U} \mathrm{L}$ peaks. Filters were used to reduce the background signal due to the pile-up from the light elements and from the $\mathrm{M}$ line signals of $\mathrm{U}$ and $\mathrm{Pu}$, and also to eliminate the noise created by the backscattered protons that reach the Si detector.

\subsection{ICP-MS and $\alpha$-particle spectrometry}

One isolated Palomares hot-particle was totally digested with a mixture of $\mathrm{HNO}_{3}$ and $\mathrm{HF}$ in a microwave oven. The obtained solution was divided into several aliquots, being one of them, without radiochemical separation, devoted to ICP-MS measurements while other was devoted to the determination of 

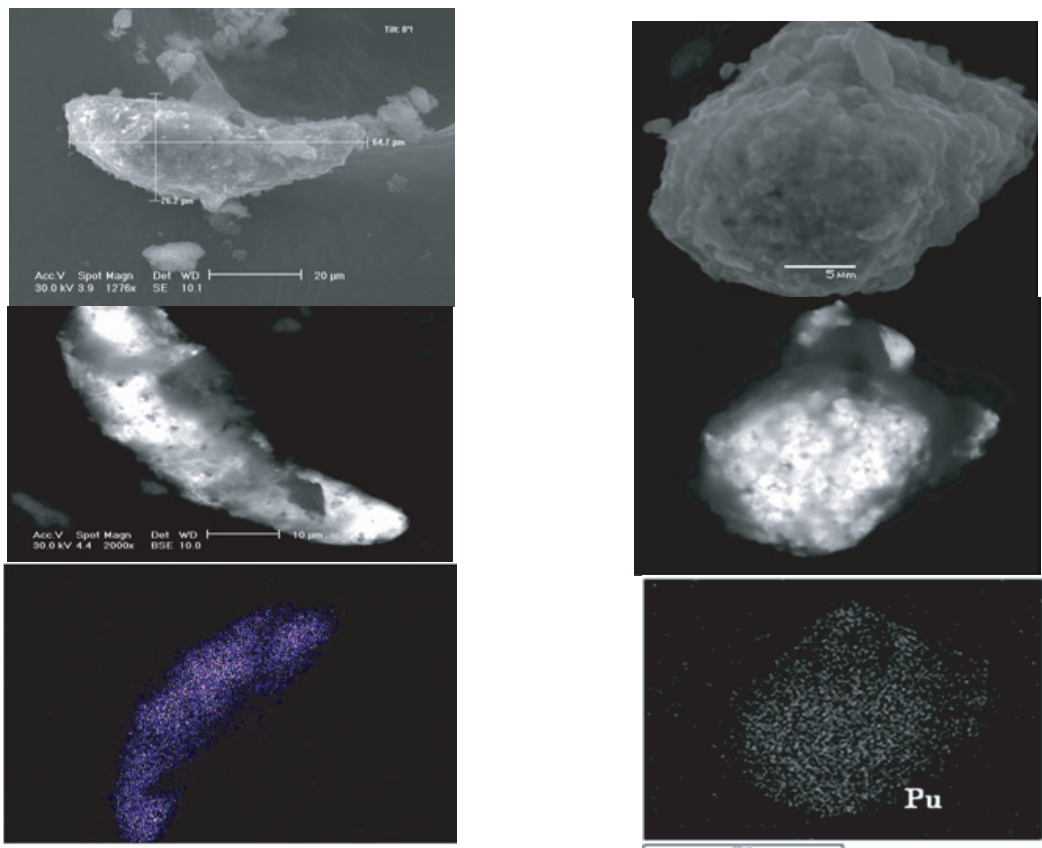

Figure 1. SEM images and XRMA Pu mappings of the two hot particles (HP3, left, and A15, right).

$\mathrm{U}$-isotopes concentrations by $\alpha$-particle spectrometry following radiochemical separation. The process of Uranium isolation and determination by $\alpha$-particle spectrometry is described in detail in [4].

A quadrupole inductively coupled mass spectrometry system was used for U-isotopes and $\mathrm{Pu}$-isotopes analysis of the sacrificed hot-particle. The system was tuned and optimized for the measurement of high mass isotopes by checking that the sensitivity for masses higher than ${ }^{235} \mathrm{U}$ was constant for the detector employed. The sensitivity was determined precisely in each set of measurements by measuring a ${ }^{238} \mathrm{U}$ calibration sample. A quite established protocol was applied with measurement of calibration and blank samples together with the aliquot of the dissolved particle to assure the quality of the obtained results.

\section{RESULTS AND CONCLUSIONS}

\subsection{SEM (EDX) and $\mu$-PIXE analyses}

In Fig. 1, images from SEM measurements are shown for the two hot particles analyzed in this work. For each particle a SE image, a BSE image and a Pu mapping (in this order form up to down) are presented.

By XRMA we have obtained the surface composition of both particles. For the first one, the surface main components are: $\mathrm{O}, \mathrm{Al}, \mathrm{Si}, \mathrm{Fe}, \mathrm{Cu}, \mathrm{U}$ and $\mathrm{Pu}$; and for the second one: $\mathrm{O}, \mathrm{Al}, \mathrm{Si}, \mathrm{Ba}, \mathrm{Fe}, \mathrm{U}$, and $\mathrm{Pu}$. It was possible to see that $\mathrm{Pu}$ and $\mathrm{U}$ are present on the surfaces of both particles while, the other elements surely, are associated to the soil coating partially the particles. In fact, the Pu mappings in both particles indicate the high association of the Pu detected in the surfaces with the brightest areas found by BSE.

In Figure 2, a typical $\mu$-PIXE spectrum corresponding to one spot in one of the hot-particles is shown. It was obtained by impinging $3 \mathrm{MeV}$ protons in the particle. The different L-lines of $\mathrm{Pu}$ and $\mathrm{U}$ can be observed (marked in red), while in the low energy region the $\mathrm{Pu}$ and $\mathrm{U} \mathrm{M}$ lines as well the 


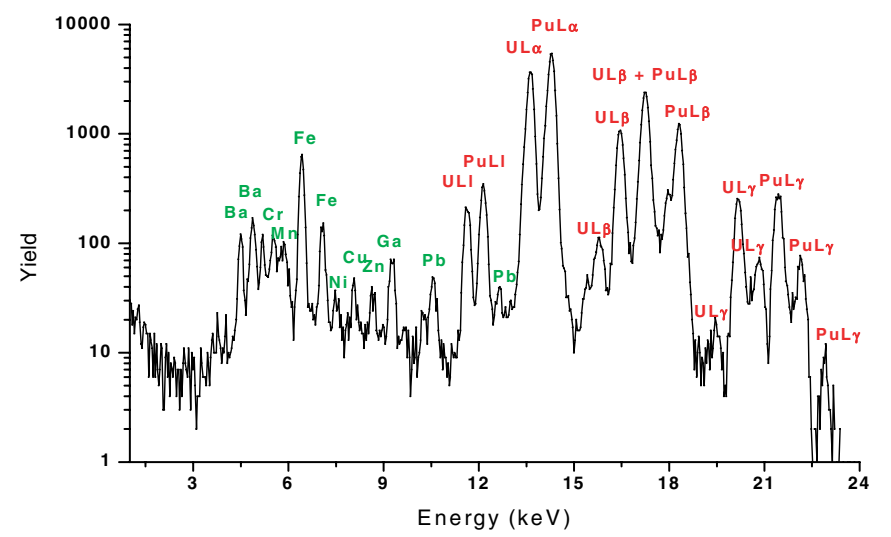

Figure 2. PIXE spectrum recorded on a point of one of the hot-particles analysed in this work.

Table 1. $\mu$-PIXE spot measurements on the particles under protons and alpha particles impingements.

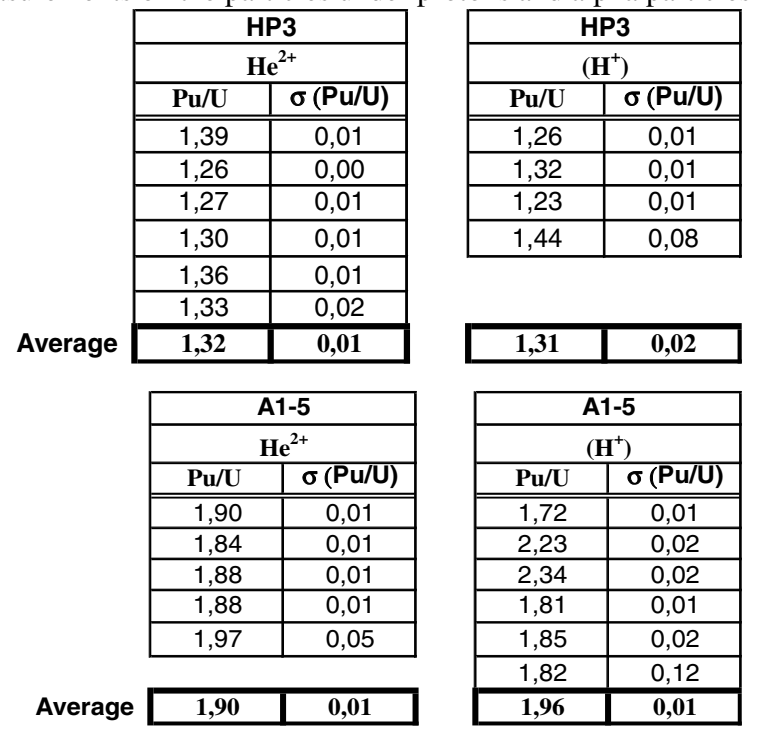

X-rays of lighter elements (marked in green) are drastically reduced due to the action of the filter placed between the particle and the detector.

After fitting the PIXE spectra and taking into account the X-ray production correction for $\mathrm{U}$ and $\mathrm{Pu}$ in both cases [1], it was possible to calculate the $\mathrm{Pu} / \mathrm{U}$ elemental ratios. The $\mathrm{Pu} / \mathrm{U}$ ratios obtained by $\mu$-PIXE with protons and alpha particles are compiled in Table 1 and are given for different spots in each particle. For HP3 particle, the ratios are very similar for all the measurements with independence of the beam used, indicating that the $\mathrm{Pu} / \mathrm{U}$ atom ratio homogeneity is appreciable in its composition. However, in the A1-5 particle, the proton data point out different values for the $\mathrm{Pu} / \mathrm{U}$ ratios along the particle, whereas in alpha particle analysis the ratios are clearly more homogeneous. These facts indicate the possible existence in the A1-5 particle of some heterogeneity in the Pu/U distribution with depth.

If, in more detail, we compare on the A1-5 particle only the proton and alpha particle results obtained for spots located in the same place (Table 2), we could confirm if in these points the $\mathrm{Pu} / \mathrm{U}$ ratios near the surface and in the whole particle have similar values or not. Looking to the data of the mentioned table, it is possible to confirm the heterogeneity of the $\mathrm{Pu} / \mathrm{U}$ elemental ratio with depth, because this ratio has a higher value inside the particle. 
Table 2. Comparison between $\mu$-PIXE measurements (protons and $\alpha$-particles) in same spots on A1-5 particle.

\begin{tabular}{|c|c|c|c|}
\hline \multicolumn{4}{|c|}{$\mathbf{A 1 - 5}$} \\
\hline \multicolumn{2}{|c|}{$\mathbf{H e}^{2+}$} & \multicolumn{2}{c|}{$\left(\mathbf{H}^{+}\right)$} \\
\hline \hline $\mathbf{P u} / \mathbf{U}$ & $\boldsymbol{\sigma}(\mathbf{P u} / \mathbf{U})$ & $\mathbf{P u} / \mathbf{U}$ & $\boldsymbol{\sigma}(\mathbf{P u} / \mathbf{U})$ \\
\hline \hline 1,88 & 0,01 & 2,23 & 0,02 \\
\hline 1,88 & 0,01 & 2,34 & 0,02 \\
\hline
\end{tabular}

Finally, the comparison of the $\mathrm{Pu} / \mathrm{U}$ ratios gained by $\mu$-PIXE with the obtained by SEM-EDX measurements for the particle A1-5 (which gives only surface information), reinforce the previously obtained conclusion about the heterogeneity of the $\mathrm{Pu} / \mathrm{U}$ elemental ratios with depth in this particle. The average $\mathrm{Pu} / \mathrm{U}$ ratio obtained by SEM-EDX (superficial ones) in a set of 6 spots on A1-5 particle is 1.62, clearly lower than the obtained ones by $\mu$-PIXE with protons, and even lower than the obtained ones impinging on the hot-particle alpha particles. All the facts allow concluding that there is an increasing gradient of the $\mathrm{Pu} / \mathrm{U}$ elemental ratio $t$ in the $\mathrm{A} 1-5$ particle from the surface to deeper zones.

\subsection{ICP-MS and $\alpha$-particle spectrometry analyses}

The ICP-MS measurements performed in one aliquot of the sacrificed hot-particle permitted the determination of their ${ }^{238} \mathrm{U},{ }^{235} \mathrm{U},{ }^{239} \mathrm{Pu}$ and ${ }^{240} \mathrm{Pu}$ levels (see Table 3). The corresponding ICP-MS spectrum is shown in Figure 3.

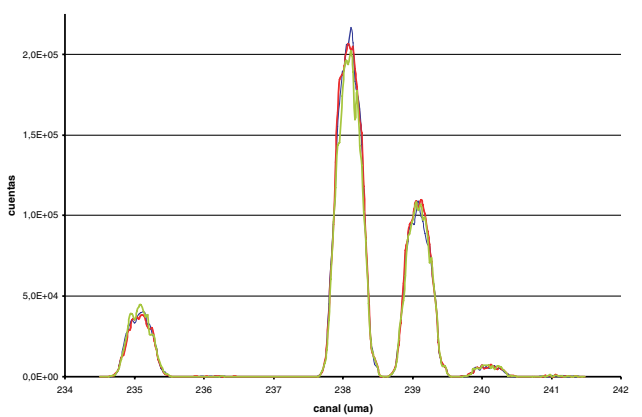

Figure 3. ICP-MS mass spectrum corresponding to the analyzed hot-particle.

Table 3. ${ }^{238} \mathrm{U},{ }^{239} \mathrm{Pu}$ and ${ }^{240} \mathrm{Pu}$ levels and ${ }^{238} \mathrm{U} /{ }^{235} \mathrm{U}$ and ${ }^{240} \mathrm{Pu} /{ }^{239} \mathrm{Pu}$ atom ratios in one hot-particle.

\begin{tabular}{|c|c|c|c|c|}
\hline $\begin{array}{c}{ }^{238} \mathbf{U} \\
(\mathbf{n g})\end{array}$ & $\begin{array}{c}{ }^{239} \mathbf{P u} \\
(\mathbf{n g})\end{array}$ & $\begin{array}{c}{ }^{240} \mathbf{P u} \\
(\mathbf{n g})\end{array}$ & $\begin{array}{c}{ }^{238} \mathbf{U} /{ }^{235} \mathbf{U} \\
\text { atom ratio }\end{array}$ & $\begin{array}{c}{ }^{240} \mathbf{P u} /{ }^{239} \mathbf{P u} \\
\text { atom ratio }\end{array}$ \\
\hline $16.0 \pm 2.3$ & $8.3 \pm 0.4$ & $0.53 \pm 0.02$ & $5.4 \pm 0.2$ & $0.063 \pm 0.003$ \\
\hline
\end{tabular}

The results show a ${ }^{238} \mathrm{U} /{ }^{235} \mathrm{U}$ atom ratio clearly lower than the found one in the nature and a quite low ${ }^{240} \mathrm{Pu} /{ }^{239} \mathrm{Pu}$ atom ratio. These facts give a strong indication that the nuclear weapons involved in the Palomares accident contained a mixture of enriched uranium and weapon grade plutonium.

The presence of enriched uranium in the Palomares hot-particles was also confirmed by isolating the uranium content from one of the aliquots of the sacrificed hot-particle, by electroplating the isolated $\mathrm{U}$ on a stainless-steel planchet and, finally, by measuring it by $\alpha$-particle spectrometry. The obtained spectrum is shown in Figure 4. In this $\alpha$-spectrum is possible to observe the enormous disequilibrium between ${ }^{234} \mathrm{U}$ and ${ }^{238} \mathrm{U}\left({ }^{234} \mathrm{U} /{ }^{238} \mathrm{U}>100\right)$ typically found in enriched uranium, and the presence of ${ }^{235} \mathrm{U}$ activity levels higher than the found ones for ${ }^{238} \mathrm{U}$. 


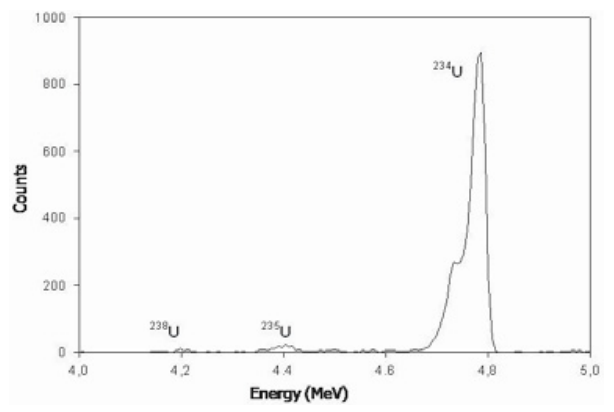

Figure 4. Uranium $\alpha$-spectrum corresponding to the analyzed hot particle.

$\mathrm{The} \mathrm{Pu} / \mathrm{U}$ atom ratio which can be calculated from the ICP-MS is lower than the determined ones by $\mu$-PIXE and even by XRMA. The reason is clear: A total isolation of the sacrificed hot particle was not possible due to the difficulties in the removal of some soil material coating it. This fact introduces some natural ${ }^{238} \mathrm{U}$ associated to the coated material in the analysed solutions which shift the real $\mathrm{Pu} / \mathrm{U}$ atom ratio of the hot particle to lower values. Nevertheless, due to the particular $\mathrm{U}$ isotopic content of the particle, the ${ }^{235} \mathrm{U}$ and ${ }^{234} \mathrm{U}$ levels determined by the destructive techniques are practically not affected by the simultaneous digestion of the coating soil material.

\section{Acknowledgments}

This work has been supported by the Junta de Andalucía Excellence Project TU-DRAMA (Ultra-sensitive techniques for radionuclides determination in environmental materials).

\section{References}

[1] J. García López, M.C. Jiménez-Ramos, M. García-León, R. García-Tenorio, Nucl. Instr. and Meth. $B$ 260, 343 (2007).

[2] M.C. Jiménez-Ramos, H. Barros, R. García-Tenorio, M. García-León, I. Vioque, G. Manjón, Environmental Pollution 145, 391 (2007).

[3] M.C. Jiménez-Ramos, R. García-Tenorio, I. Vioque, G. Manjón, M. García-León, Environmental Pollution 142, 487 (2006).

[4] E. Holm, R. Fukai, Talanta 24, 659 (1977). 